\title{
Probing structural relaxation in nanosized catalysts by combining EXAFS and reverse Monte Carlo methods
}

\author{
Janis Timoshenko ${ }^{\mathrm{a}, *}$, Anatoly I. Frenkel ${ }^{\mathrm{a}, *}$ \\ ${ }^{a}$ Department of Physics, Yeshiva University, New York, New York 10016, United States
}

\begin{abstract}
Strain-induced structure relaxation, large structural disorder and complex nonGaussian shape of the interatomic distances distribution are characteristic even for the simplest monometallic nanocatalysts, and may have significant impact on many properties, including catalytic ones. Extended X-ray absorption fine structure (EXAFS) spectroscopy is able to provide unique information on the structure of nanosized catalysts due to its sensitivity to short-range order in the material. The EXAFS studies of disorder effects in small nanoparticles (with size 1 - $2 \mathrm{~nm}$ ) are, however, challenging due to the assumption of quasiGaussian bond length distributions that is made in the conventional analysis. One possible solution shown in this study for bare gold nanoparticles is a novel advanced approach: EXAFS data modelling using reverse Monte Carlo and evolutionary algorithm methods. In addition, we use here classical molecular dynamics simulations to validate the obtained results.
\end{abstract}

Keywords: gold nanoparticles, EXAFS, molecular dynamics, reverse Monte Carlo, evolutionary algorithm

PACS: 61.05.cj, 78.70.Dm, 61.46.Df, 02.70.Ns

\footnotetext{
${ }^{*}$ Corresponding author

Email addresses: : janis.timoshenko@gmail.com (Janis Timoshenko), : anatoly.frenkel@yu.edu (Anatoly I. Frenkel)
}

Preprint submitted to Catalysis Today

May 19, 2016

(C) 2016. This manuscript version is made available under the Elsevier user license http://www.elsevier.com/open-access/userlicense/1.0/ 


\section{Introduction}

Many applications of metallic nanoparticles (NPs), in particular in catalysis, rely on the observation that properties (magnetic, optical, chemical, catalytic) of such materials are different from those of bulk material, and can be tailored

5 by changing the NPs composition, size and shape [1, 2, 3, 4]. The properties and potential practical applications of NPs depend on their atomic structure, which can be very different from that in bulk material. Finite size effects, interactions with support and/or adsorbates distort the periodic structure in NPs, resulting in significant deviations of atom arrangements in small NPs from close-packed structure of bulk metal [5, 6, 7, 8, As a result, much larger static disorder is expected. Many such effects result in broad and asymmetric distributions of interatomic distances 9 , 10, 11, 12, 13.

At the same time the choice of tools to investigate the local structure in small $(1-2 \mathrm{~nm})$ NPs is rather limited [14], since the common crystallographical approaches are not applicable in this case and the sensitivity of high resolution electron microscopy methods is not sufficient to resolve in details the atomic structure of such small NPs. One of the methods that, nevertheless, allows one to probe the complex structure and dynamics of nanosized materials, is X-ray absorption spectroscopy. In particular, analysis of extended X-ray absorption fine structure (EXAFS) can be useful, since it encodes the information on the configuration-averaged arrangements of atoms around the absorbing atoms [15]. Providing unique information about interatomic distances and types of neighboring atoms, EXAFS spectroscopy has been applied to the investigations of structure of nanosized catalysts for decades [16, 17.

There are several challenges associated with the EXAFS analysis of nanocrystalline materials. Importantly, conventional EXAFS analysis requires to assume a priori some shape for the distribution of interatomic distances. Parameters 
of this distribution (e.g., average interatomic distances and variances) are then obtained by fitting theoretical signal to experimental EXAFS spectrum [15, 18].

30 Most frequently used harmonic approximation assumes Gaussian distribution of bond lengths, and is usually adequate for the description of bulk materials. However, as pointed above, the distributions of interatomic distances in metallic NPs can be much more complicated. The use of a Gaussian model for bond lengths distribution results in this case in unphysical values of estimated struc35 ture parameters 9, 12. There are known efforts to correct for the asymmetric shape of distance distribution by modelling it with skewed Gaussian [19, 20] or even asymmetric $\Gamma$ function [10, 21, 22, but these approaches, while they may work in certain specific cases, cannot be considered as a general solution. Another possible approach is to perform model-free fitting of bond lengths distri40 bution using regularization-like techniques [23, 24], but usually they are limited to the analysis of the first coordination shell only. In fact, the limited ability to analyze the disorder in material structure beyond the first coordination shell is an issue for conventional EXAFS studies in general, and not only for NPs, but even for bulk material, since contributions of different coordination shells 45 overlap with multiple scattering contributions [10, 25].

On the other hand, in the last decade it was demonstrated that the use of theoretical modelling approaches allows one to fill in the gaps in the data, provided by experimental techniques [14, 26]. The use of ab initio simulations within density functional theory (DFT) formalism allows one to model relaxation processes in metallic NPs, and can be successfully used for prediction of properties and structure of nanosized catalysts [27, 28, 29]. Molecular dynamics (MD) simulations using interatomic forces that are derived either from ab initio calculations [12, 30] or from empirical force field model [21, 26, 31, 32] can be used, in turn, to model thermal disorder effects and anharmonic motion of 
atoms. Thus theoretical modelling can be a very powerful tool to advance our understanding of local structure and dynamics in metallic NPs, especially if the findings of simulations can be validated using experimental approaches, such as EXAFS analysis.

Nevertheless, there are still some limitations in the accuracy and applicability of DFT and MD approaches. In particular, ab initio calculations are computationally very demanding for such distorted systems as metallic NPs, and are currently limited to investigations of NPs not larger than $1-2 \mathrm{~nm}$ [26. Classical MD simulations (simulations with empirical force field models), in turn, are believed to be less accurate, especially when the interactions with ligands and/or support are important. Note also, that neither ab initio MD, nor classical MD cannot be applied to simulations at low temperature, since zero point vibrations due to quantum effects are not taken into account in conventional MD calculations.

As an attractive alternative to DFT and MD simulations in this paper we employ reverse Monte Carlo (RMC) modelling [33. RMC method can be considered as a crossover between conventional EXAFS fitting and theoretical modelling approaches. Similarly to conventional EXAFS analysis, RMC approach is a fitting procedure, that is guided by experimental data and does not require DFT simulations or knowledge of force field model. Hence it is a very general method that can be used for investigations of a broad range of materials within a broad range of experimental conditions 34. In this case, the fitting variables are atomic coordinates within 3D atomic configuration. Therefore as a result of RMC simulations we obtain a complex 3D structure model of the material, which can be compared directly with the results of DFT or MD simulations. Structure parameters of interest can be calculated from coordinates of atoms in the RMC model without any assumptions on the shape of bond length distribu- 
tions. While RMC simulations typically require more computational resources than classical MD simulations, they are far less computationally demanding than ab initio MD modelling, especially when RMC calculations are coupled with recently proposed evolutionary algorithm (EA) scheme for more efficient structure model optimization [35]. RMC/EA approach allows us also to account for and benefit from the contributions of distant coordination shells and multiple-scattering effects. However, as for any fitting procedure, the structure model, obtained in RMC/EA simulations, can be only as good as the experimen90 tal data used. Therefore for each particular case the applicability of RMC/EA simulations needs to be validated, and one needs to estimate systematic errors of the analysis. For the validation one can carry out RMC/EA calculations using model EXAFS data instead of experimental spectrum. Such model data can be generated, e.g., in MD simulations, and we can use the same approach 95 as employed in [9, 12] to probe the limits of conventional EXAFS analysis.

The feasibility of RMC/EA approach for EXAFS data analysis have been recently demonstrated by us in studies of bulk crystalline oxide materials [35, 36] and in studies of nanostructured tungstates with very small nanocrystalites consisting of just a few coordination polyhedra [37. Here for the first time we apply this approach to studies of structure in strained metal nanoparticles, with the aim to recover and investigate the asymmetric distributions of interatomic distances. For reliable analysis, this complicated problem will be addressed in several steps. First, we will validate the RMC/EA-EXAFS method using model $\mathrm{Au} \mathrm{L}_{3}$-edge EXAFS data, obtained in classical MD simulations for bulk gold and for gold nanoparticles of about $2 \mathrm{~nm}$ in size. Next, we will use the presented method to reconstruct the radial distribution of atoms from the experimental $\mathrm{Au}$ $\mathrm{L}_{3}$-edge EXAFS data for gold foil. Finally, we will discuss the results obtained

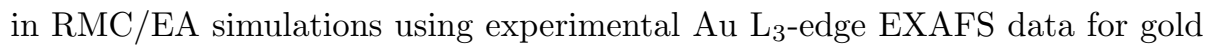


nanoparticles, synthesized using dendrimer-encapsulation technique [12].

\section{Material, methods and calculations}

\subsection{Samples and EXAFS data}

Experimental $\mathrm{Au} \mathrm{L}_{3}$-edge EXAFS data for gold foil and bare gold nanoparticles that were synthesized using dendrimer-encapsulation technique [38] were taken from [12. All EXAFS measurements have been performed at room temperature.

Using scanning transmission electron microscopy (STEM) it was verified in [12] that all gold nanoparticles have approximately the same spherical shape and size, with diameter $1.7 \pm 0.3 \mathrm{~nm}$. The STEM findings are consistent with the assumption that investigated particles contain on average 147 gold atoms, the magic number for both icosahedral and cuboctahedral cluster series. Such structural monodispersity of synthesized NPs, provided by dendrimer-encapsulation technique, allows us to employ advanced EXAFS analysis approaches and compare directly the results of EXAFS analysis with the results of theoretical modelling [27, 38].

Experimental EXAFS data and their Fourier transforms (FTs) for gold foil and gold nanoparticles are shown in Fig. 1. Data analysis by conventional fitting approaches (FEFFIT code [18]) have been presented in [12.

\subsection{Reverse Monte Carlo and evolutionary algorithm approach.}

In this study to treat $\mathrm{Au}_{3}$-edge EXAFS data we employ reverse Monte Carlo approach, complemented with evolutionary algorithm method for more efficient structure model optimization [35. The combined RMC/EA scheme allows us (i) to perform accurate analysis of EXAFS data without any assumptions about the shape of interatomic distances distribution, (ii) to take into account contributions from distant coordination shells and multiple scattering 
phases and amplitudes on relative positions of atoms. Clearly, all these peculiarities of $\mathrm{RMC} / \mathrm{EA}$ analysis are important for the analysis of nanostructured materials, where disorder effects are typically larger than in bulk material due to structure relaxation, but where contribution of outer coordination shells still may be important.

In RMC/EA approach we vary the coordinates of atoms in a sufficiently large 3D model of investigated material, with the goal to find such atomic configuration, for which the calculated theoretical configuration-averaged EXAFS spectrum will give the best possible agreement with experimental data. During the simulations, the initial structure model is improved in an iterative stochastic process. As in the conventional RMC procedure [33] at each iteration a new atomic configuration is proposed, by random displacing all atoms in the configuration. For the new configuration then corresponding ab initio configuration-averaged EXAFS spectrum is calculated. The proposed configuration is then evaluated, by comparing the calculated spectrum with the experimental one. Next, one decides, if the proposed modification should be accepted or the model should be returned to the previous state. For this purpose Metropolis algorithm [39] is used, which accepts all proposed configurations that improve the agreement between experimental and theoretical EXAFS, and also some of the proposed configurations that slightly worsen the agreement. Such approach ensures proper sampling of the configurational space and allows one to avoid being trapped in some local minimum. In our case we also employ the so called simulated annealing approach [40, where at the beginning of RMC run almost all proposed moves are accepted, but then the acceptance ratio for configurations that worsen the agreement between theory and experiment is gradually reduced. At the end of simulations only moves that improve the agreement between experiment and 
theory are accepted. After such improvement is no longer possible, the final atomic configuration then is considered as the one that gives the best possible agreement with experiment.

Implementation of the evolutionary algorithm [41, 42] in the conventional RMC scheme, described above, significantly reduces the number of required iterations, hence it allows to analyze more complex material structures with the same computational resources available. In our case the use of evolutionary algorithm simply means that we are simulating not just one, but several (64 to 128 in this case) atomic configurations in parallel, and we allow information exchange between them. At each iteration from the old set of atomic configurations a new set is generated (i) by copying from the old set the configurations that gave the best agreement with experiment to the new set, (ii) by interchanging some atoms of randomly selected configuration with atoms of another 175 configuration and (iii) by shifting all atoms in all configurations in random directions, as described above. The details of RMC/EA procedure are described in [35] and [43].

In this paper we apply RMC/EA approach to analyze $\mathrm{Au} \mathrm{L}_{3}$ EXAFS data for bulk gold foil and Au147 nanoparticles. In the case of bulk gold, the initial structure model is face-centered cubic (fcc) structure with lattice parameter $a_{0}=4.080 \AA$ [26]. For our simulations we use $6 \times 6 \times 6$ supercell containing 864 gold atoms and employ periodic boundary conditions to avoid surface effects. In our RMC/EA calculations for gold nanoparticles as a starting model we use gold cluster with 147 gold atoms, and we test two different geometries with (i) 185 cuboctahedral and (ii) icosahedral shapes. Moreover, since the experimental EXAFS data are averaged over large number of particles and over long period of time, the actual structure model that we optimize in our simulations is also not a single particle, but a small ensemble of such particles: we have found that 
an ensemble consisting of 8 nanoparticles is sufficient to fit experimental data.

In this study both for bulk gold and gold NPs the overall shape and topology of the model is maintained constant during the simulations, by constraining the maximal allowed displacements of atoms from their initial positions to be smaller than $0.5 \AA$.

At each RMC/EA iteration for each gold atom in the configuration EXAFS spectrum is calculated using ab initio self-consistent real space multiplescattering FEFF8.5L code [44, taking into account single scattering, double scattering and triple scattering paths with half lengths up to $6.5 \AA$. Thus about 700 paths per each absorbing atom are used in calculations for bulk material, and, on average, about 200 paths per atom in calculations for nanoparticles.

The same self-consistent cluster potential, calculated for equilibrium bulk gold structure, is used for all atoms during the simulations, thus neglecting its small variation due to thermal and static disorder. The complex exchangecorrelation Hedin-Lundqvist potential and default values of muffin-tin radii are employed. The dependency of scattering functions (phase and amplitude) on actual relative positions of atoms with respect to the absorbing atom is taken into account explicitly, since the scattering amplitudes and phases are recalculated for each scattering path in each atomic configuration. Configuration-averaged EXAFS spectrum that can be compared with experimental data is then obtained by averaging contributions of all absorbing Au atoms in the structure model.

The comparison of calculated spectra with experimental data is carried out using continuous Morlet wavelet transform [45, 46, as discussed in [47, 35, in the $k$-range from $2.4 \AA$ up to $14 \AA$ and in the $R$ range from $1.0 \AA$ up to $6.0 \AA$.

The accuracy of RMC modelling is always limited by the information amount, ${ }_{215}$ available from experimental spectra. Especially important this issue is for the 

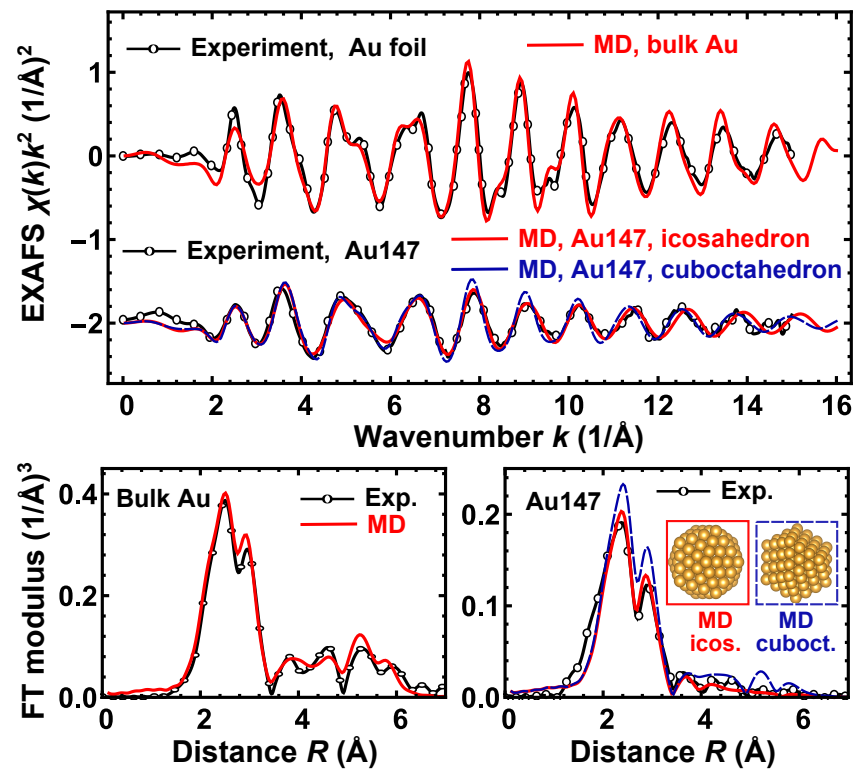

Figure 1: (Color online) Au $\mathrm{L}_{3}$-edge EXAFS spectra for bulk gold and gold nanoparticles (Au147). Experimental EXAFS spectra for gold foil and Au147 NPs and calculated configuration-averaged EXAFS spectra, obtained in classical MD simulations with SuttonChen force field model, are shown together with their Fourier transforms (FTs). For gold NPs we compare two MD-EXAFS spectra that were obtained in calculations with two different geometries: icosahedral and cuboctahedral.

analysis of EXAFS data for strongly distorted materials, where static disorder reduces significantly the contribution of outer coordination shells to the total spectrum. Therefore, to estimate the systematic errors of the analysis, in this study we apply RMC/EA simulations not only to experimental EXAFS data, but also to fit model EXAFS data, for which the corresponding structure is already known. To generate such model data, we employ classical molecular dynamics simulations.

\subsection{Molecular dynamics simulations}

For classical MD simulations for bulk gold and gold nanoparticles, we use Sutton-Chen type force field model [48].

The potential energy of the system $U$ is defined in this model as 


$$
U=\sum_{i}\left[\sum_{j \neq i} \frac{1}{2} \varepsilon\left(\frac{a}{r_{i j}}\right)^{n}-c \varepsilon\left(\rho_{i}\right)^{\frac{1}{2}}\right],
$$

where

$$
\rho_{i}=\sum_{j \neq i}\left(\frac{a_{0}}{r_{i j}}\right)^{m},
$$

$a_{0}$ is gold lattice constant, $r_{i j}$ denotes distance between $i$-th and $j$-th atom and $m, n, \varepsilon$ and $c$ are empirical parameters. The values of these parameters were taken from [26] and are summarized in Table 1. They were chosen to reproduce structure and elastic properties of bulk gold material. The accuracy of this force field model for simulations of large $(4-6 \mathrm{~nm})$ gold nanoparticles was demonstrated in [26] by comparing results of MD simulations with the results of total scattering experiments (pair distribution function analysis). Note that for 235 the interpretation of EXAFS spectra in metallic NPs similar force-field model was employed in 32 for investigations of supported platinum nanoparticles.

Table 1: Values of Sutton-Chen force-field parameters that were used for classical MD simulations [26].

\begin{tabular}{ccccc}
\hline$m$ & $n$ & $\varepsilon(\mathrm{meV})$ & $a(\AA)$ & $c$ \\
8 & 11 & 7.8052 & 4.080 & 53.581 \\
\hline
\end{tabular}

MD simulations are performed using GULP code [49, 50, in the canonical (NVT) ensemble. For simulations of bulk gold we employ periodic boundary conditions and the simulation box has a size of the $5 a_{0} \times 5 a_{0} \times 5 a_{0}$ supercell including 500 atoms. For simulations of Au147 nanoparticles two different starting models for gold nanoparticles were constructed: with (i) cuboctahedral and (ii) icosahedral geometries.

Nosé-Hoover thermostat [51] is used to keep the required average temperature $(T=300 \mathrm{~K})$ during each simulation. The Newton's equations of motion ${ }_{245}$ are integrated using the Verlet leapfrog algorithm [52] with a time step of $0.5 \mathrm{fs}$. 
The equilibration time is $20 \mathrm{ps}$, followed by 20 ps of production run, during which 4000 atomic configurations are accumulated.

For each of the 4000 configurations a theoretical EXAFS spectrum, averaged over all absorbing atoms, is calculated using FEFF8.5L 44] software. For this purpose the same approach is used here as in calculations of EXAFS spectra for RMC/EA simulations (see Section 2.2). Calculated configuration-averaged spectra are then averaged over time, and the final time- and configurationaveraged spectrum (MD-EXAFS) can be then directly compared with experimental data [53, 54] (see Fig. 1). This model spectrum can also be used to validate our RMC/EA approach. For this purpose we carry out RMC/EA simulations using MD-EXAFS spectrum instead of experimental spectrum to guide the simulations. The final structure, obtained after RMC/EA fitting, then can be compared with the known structure of MD model, and the statistical errors of the RMC/EA analysis can be estimated. Note that we use the same calculation parameters and the same ranges in $k$ and $R$ space both for the analysis of experimental and of model EXAFS data.

Note also that calculations of MD-EXAFS spectra require less computational resources than consequent RMC/EA analysis. MD simulations (generation of atomic trajectories), considered in this paper, requires just a few hours on a 265 single CPU computer. Calculations of EXAFS spectra for the MD-generated atomic configurations are computationally more expensive, and within our approach required several hours of calculations on a computer with $16 \mathrm{CPU}$ available. Each of the RMC/EA simulations, discussed in this paper, in turn, required about 7 - 9 days of computations on a computer with $16 \mathrm{CPU}$ cores available. 


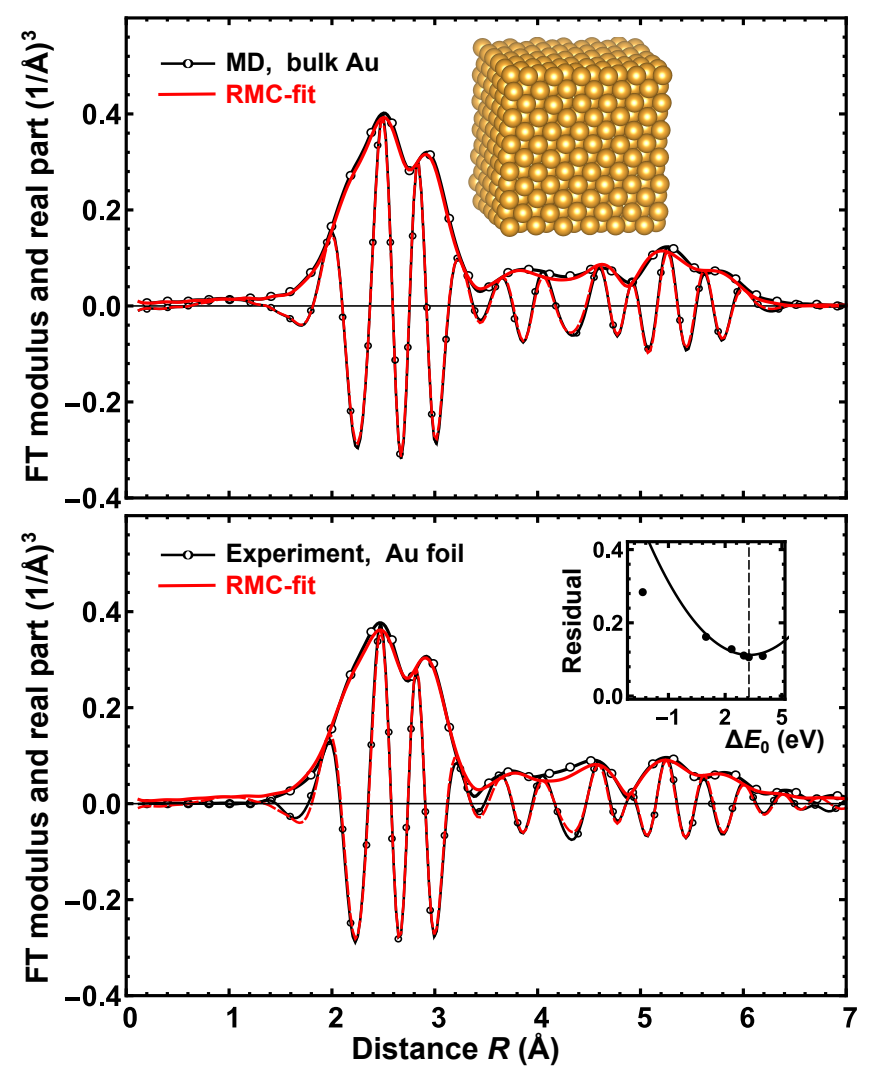

Figure 2: (Color online) Results of RMC/EA fitting for bulk gold MD model data and for experimental $\mathrm{Au} \mathrm{L}_{3}$-edge EXAFS for gold foil: moduli and real parts of Fourier transforms. The final configuration obtained in RMC/EA calculations using model data is shown in the inset of upper panel. In the inset of bottom panel - dependency of the final difference between experimental and simulated EXAFS on the used value for the correction to photoelectron energy origin $\Delta E_{0}$.

\section{Results and discussion}

\subsection{MD model for bulk gold}

Experimental $\mathrm{Au} \mathrm{L}_{3}$-edge EXAFS spectrum for gold foil and MD-EXAFS spectrum for bulk gold are compared in Fig. 1 Both spectra are in a good agreement, which is especially remarkable, since there were no fitted parameters in our MD simulations. The only exception were the two non-structural EXAFS parameters: amplitude reduction factor $S_{0}^{2}$ and the correction $\Delta E_{0}$ to the photoelectron energy origin $E_{0}\left[15\right.$. The values of $S_{0}^{2}=0.93$ and $\Delta E_{0}=2.27 \mathrm{eV}$ 
were obtained from the conventional fitting of the contribution of the first coorcode [18] was employed. The good agreement between experiment and theory indicates that local structure of gold can be reasonably reproduced by SuttonChen force field model. It also shows us that the disorder in the MD model and in the real gold material are similar, hence RMC/EA simulations using model EXAFS spectrum can be reliably used to estimate uncertainties of RMC/EA analysis for gold foil.

Results of RMC/EA fitting of MD-EXAFS data and experimental data for bulk gold are shown in Fig. 2 For model data, RMC/EA approach provides almost perfect fit in all $R$-range, used for calculations. The final structure model, obtained in the calculations using bulk gold model data, is shown in the inset in the upper panel of Fig. 2. We can use now the atomic coordinates, obtained in RMC/EA simulations, to calculate structure parameters of interest, and to compare them with the values of these parameters, calculated directly from MD coordinates.

Radial distribution function (RDF) $g(R)$ is defined as probability density to find an atom at distance $R$ from the absorbing atom. In our case we normalize it so that integral

$$
N_{i}=\int_{r_{\min }^{i}}^{r_{\max }^{i}} g(r) d r
$$

correspond to an average number of atoms $N_{i}$ at distances between $r_{\min }^{i}$ and $r_{\max }^{i}$ from the absorbing atom. $N_{i}$ corresponds to average coordination number for the $i$-th shell, if we choose $r_{\min }^{i}$ and $r_{\max }^{i}$ so that all atomic pairs corresponding to the $i$-th coordination shell in the equilibrium structure are included in the integration. Function $g(R)$, calculated from the coordinates of MD simulations, 
is compared with the results of $\mathrm{RMC} / \mathrm{EA}$ reconstruction in the upper panels of Fig. 3 The positions of RDF peaks correspond to average interatomic distances, while their widths characterize the disorder in the corresponding atomic pair. As shown in Fig. 3, for bulk material our RMC/EA approach is able to reconstruct quite accurately the radial distribution of atoms at distances at least up to $6 \AA$ from the absorbing atom. To make the comparison more quantitative, in Table 2 we list the obtained values for the average interatomic distances $R_{i}$ and mean-square relative displacement (MSRD) factors $\sigma_{i}^{2}$ for the first four $(i=1$, $2,3,4)$ coordination shells. In our case we can define $R$ as

$$
R_{i}=\frac{1}{N_{i}} \int_{r_{\min }^{i}}^{r_{\max }^{i}} g(r) r d r
$$

Similarly, the MSRD factors, which characterize the disorder in the given coordination shell, can be calculated as

$$
\sigma_{i}^{2}=\frac{1}{N_{i}} \int_{r_{\min }^{i}}^{r_{\max }^{i}} g(r)\left(r-R_{i}\right)^{2} d r
$$

The results, given in Table 2, show that in the ideal case of model data for simple bulk material, RMC/EA approach is able to recover interatomic distances and MSRD factors for the first four coordination shells with accuracy better than $0.002 \AA$ and $0.0002 \AA^{2}$, respectively. Note that this estimation covers only the systematic error due to RMC/EA fitting procedure, and does not include uncertainties due to experimental noise, background subtraction issues and imperfections of FEFF calculations, which also may limit the accuracy of the analysis, when RMC/EA approach is applied to real experimental data.

For comparison, we also show in Table 2 the results, obtained for the MDEXAFS spectrum using conventional fitting using FEFFIT program [18. Only 
the contribution from the first coordination shell is analyzed and results of two

different FEFFIT fits are given. In the first case, harmonic approximation is used, i.e., Gaussian distribution of interatomic distances is assumed. In the second case, we use quasi-harmonic approximation, which allows small deviations from Gaussian shape for RDF peak, characterized by third cumulant $C_{3}$ of interatomic distance distribution [20, 12]. As one can see, even in the case of relatively well-ordered bulk material and noise-free model data, there are noticeable differences between the values of structural parameters, calculated from MD coordinates, and their estimates from FEFFIT analysis. The inclusion of the third cumulant in the fitting allows one to improve the accuracy of interatomic distance determination. Nevertheless, the disorder factors are underestimated in the conventional FEFFIT analysis. This allows us to conclude that the use of RMC/EA approach may be beneficial even for the analysis of contribution of the first coordination shell in bulk material. The main advantage of RMC/EA analysis for bulk material, however, is that it allows the accurate analysis of contributions of further coordination shells, which overlap strongly and are also strongly influenced by multiple-scattering effects. The application of conventional fitting procedures to analyze these contributions, while not impossible [10, 25], is usually very challenging.

\subsection{Analysis of experimental Au foil data}

Result of RMC/EA fitting for experimental bulk gold EXAFS data is shown 345 in the bottom panel of Fig. 2. Agreement between RMC/EA fit and experimental data is excellent: note that this agreement is significantly better than that between experimental data and MD-EXAFS data (see Fig. 1), indicating that $\mathrm{RMC} / \mathrm{EA}$ approach is able to find more accurate distribution of interatomic distances. Also note that in our RMC/EA calculations we are not fitting the values of non-structural parameters $S_{0}^{2}$ and $\Delta E_{0}$ directly. For the analysis of 


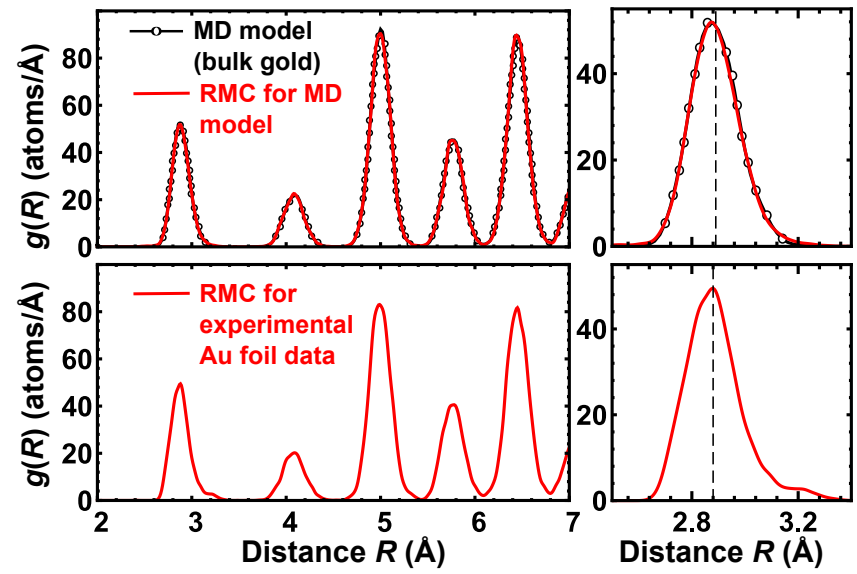

Figure 3: (Color online) Radial distribution functions $g(R)$ that were obtained in RMC/EA simulations using model bulk gold EXAFS, as well as using experimental gold foil EXAFS. For RDF that was obtained using model spectrum the RDF, calculated directly from MD simulations, is also shown for comparison. Vertical lines in the right panels denote the average interatomic distance for the first coordination shell that was calculated according to equation (4).

experimental data the same value for $S_{0}^{2}$ factor was used as mentioned above.

The value of $\Delta E_{0}$, in turn, was refined by repeating RMC/EA calculations with different values of $\Delta E_{0}$. Calculations with $\Delta E_{0}=3.26 \mathrm{eV}$ resulted in the smallest final difference between simulated and experimental spectra (see the inset in the bottom panel of Fig. 2), therefore it was used for further analysis.

RDF function that was obtained by RMC/EA fitting of gold foil data is shown in the bottom panel of Fig. 3, while the numerical values of structural parameters are summarized on the right side of Table 2. For all investigated coordination shells the average interatomic distances that were obtained from experimental $\mathrm{Au}$ foil EXAFS data and from MD data are in a good agreement. This finding emphasizes once again the remarkable accuracy of Sutton-Chen force field model. Nevertheless, our RMC/EA calculations using experimental bulk gold data suggest that larger disorder is present in the contribution of all coordination shells than it appears from MD simulations.

Deviations from Gaussian distribution are clearly visible in the shape of 
Table 2: Average interatomic distances $R_{i}$ and MSRD factors $\sigma_{i}^{2}$ for the first four coordination shells that were obtained for final RMC/EA configuration in simulations using MD-EXAFS and experimental EXAFS data for bulk gold. For comparison, the values of structure parameters that were calculated directly from molecular dynamics coordinates are given (MD), and also the results that were obtained using conventional FEFFIT fitting within harmonic $\left(\cdot{ }_{H}\right)$ and quasi-harmonic $(. \cdot \mathrm{QH})$ approximations. $C_{3}$ values, obtained in quasi-harmonic fit, are $1.8(4) \cdot 10^{-4} \AA^{3}$ for MD-EXAFS and $0.7(7) \cdot 10^{-4} \AA^{3}$ for experimental data. See details in text.

\begin{tabular}{lcccc|ccc}
\hline Parameter & MD & $\begin{array}{c}\text { FEFFIT }_{H} \\
\text { (MD-EXAFS) }\end{array}$ & $\begin{array}{c}\text { FEFFIT }_{Q H} \\
\text { (MD-EXAFS) }\end{array}$ & $\begin{array}{c}\text { RMC/EA } \\
\text { (MD-EXAFS) }\end{array}$ & $\begin{array}{c}\text { FEFFIT }_{H} \\
\text { (experiment) }\end{array}$ & $\begin{array}{c}\text { FEFFIT }_{Q H} \\
\text { (experiment) }\end{array}$ & $\begin{array}{c}\text { RMC/EA } \\
\text { (experiment) }\end{array}$ \\
\hline$R_{1}(\AA)$ & 2.889 & $2.876(2)$ & $2.888(3)$ & $2.888(1)$ & $2.858(2)$ & $2.865(5)$ & $2.889(1)$ \\
$\sigma_{1}^{2}\left(\AA^{2}\right)$ & 0.00805 & $0.0074(2)$ & $0.0074(1)$ & $0.0081(1)$ & $0.0082(2)$ & $0.0081(2)$ & $0.0094(1)$ \\
$R_{2}(\AA)$ & 4.083 & - & - & $4.082(1)$ & - & - & $4.082(1)$ \\
$\sigma_{2}^{2}\left(\AA^{2}\right)$ & 0.01153 & - & - & $0.0116(2)$ & - & - & $0.0132(1)$ \\
$R_{3}(\AA)$ & 5.000 & - & - & $4.998(1)$ & - & - & $4.998(1)$ \\
$\sigma_{3}^{2}\left(\AA^{2}\right)$ & 0.01052 & - & - & $0.0106(3)$ & - & - & $0.0125(1)$ \\
$R_{4}(\AA)$ & 5.772 & - & - & $5.771(1)$ & - & - & $5.771(1)$ \\
$\sigma_{4}^{2}\left(\AA^{2}\right)$ & 0.01052 & - & - & $0.0103(1)$ & - & - & $0.0132(1)$ \\
\hline
\end{tabular}

bond length distribution for the first coordination shell, obtained from RMC/EA analysis of experimental EXAFS for gold foil (Fig. 3). As a result, when conventional FEFFIT fitting is applied, both harmonic and quasi-harmonic approximations yield a slightly smaller value for nearest neighbor distance $(2.86$ $\AA)$ than in RMC/EA simulations $(2.89 \AA)$. Note that the former value (shown by conventional EXAFS fitting) is a subject of well-known controversy, since it is in a disagreement with available diffraction data. Note that diffraction measurements show that equilibrium distance between two nearest gold atoms is $R_{\text {eq }}=a_{0} / \sqrt{2}=2.88 \AA$, and it is known that the average bond length $R$, as probed by EXAFS, cannot be shorter than this value [55, 56]. The results, obtained by our RMC/EA analysis, are able to provide a plausible explanation to this contradiction.

\subsection{Structure of gold nanoparticles}

MD-EXAFS spectra that were obtained in MD simulations for Au147 nanoparticles are compared with experimental data in Fig. 1] Both tested initial geometries - cuboctahedral and icosahedral - appear to be stable within Sutton-Chen 


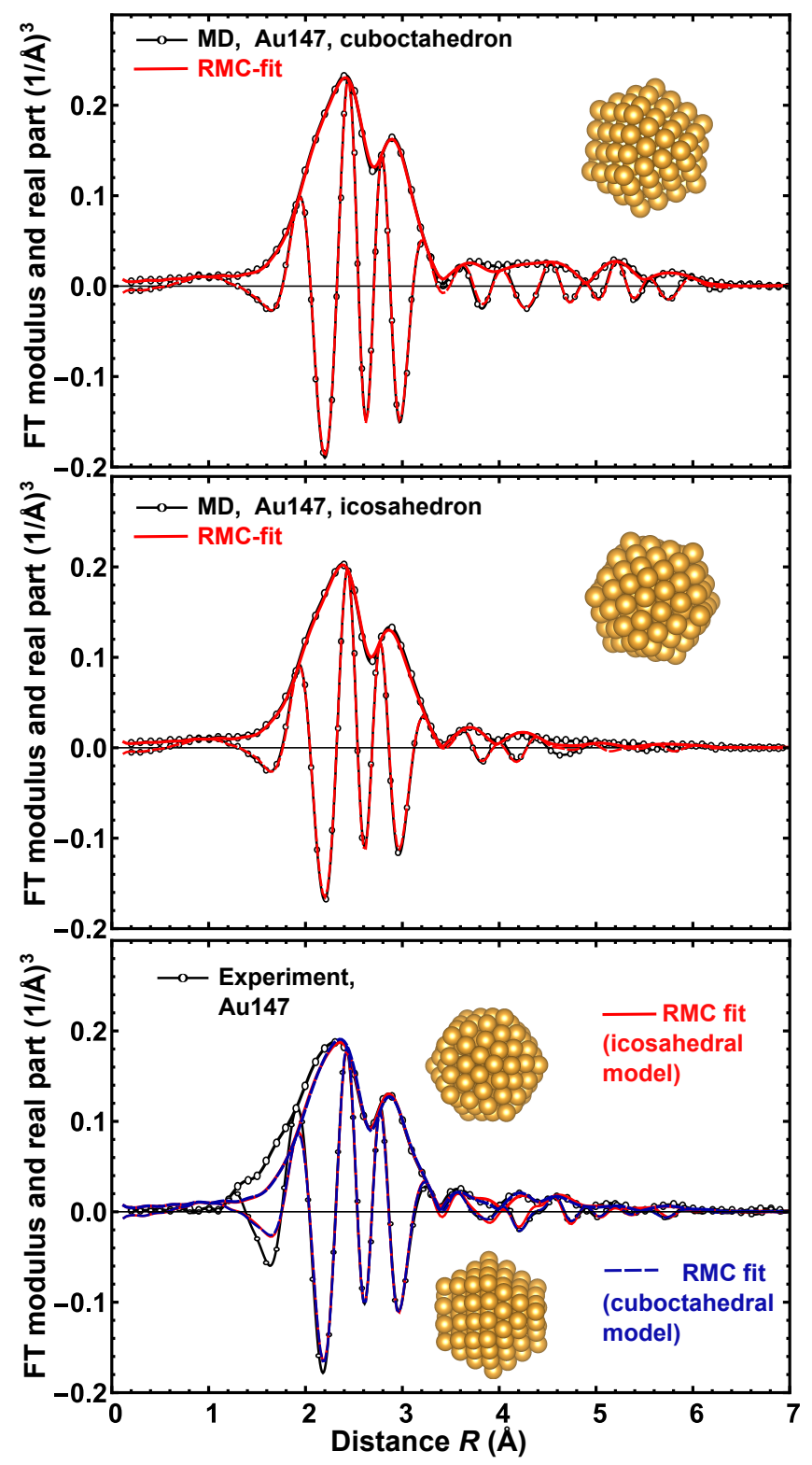

Figure 4: (Color online) Results of RMC/EA fitting for Au147 MD model data and for experimental $\mathrm{Au} \mathrm{L} \mathrm{L}_{3}$-edge EXAFS for gold nanoparticles: moduli and real parts of Fourier transforms. For experimental data the results that were obtained with different structure models (icosahedral and cuboctahedral) are compared. The representative particles from final configurations, obtained in RMC/EA calculations, are shown the insets. 


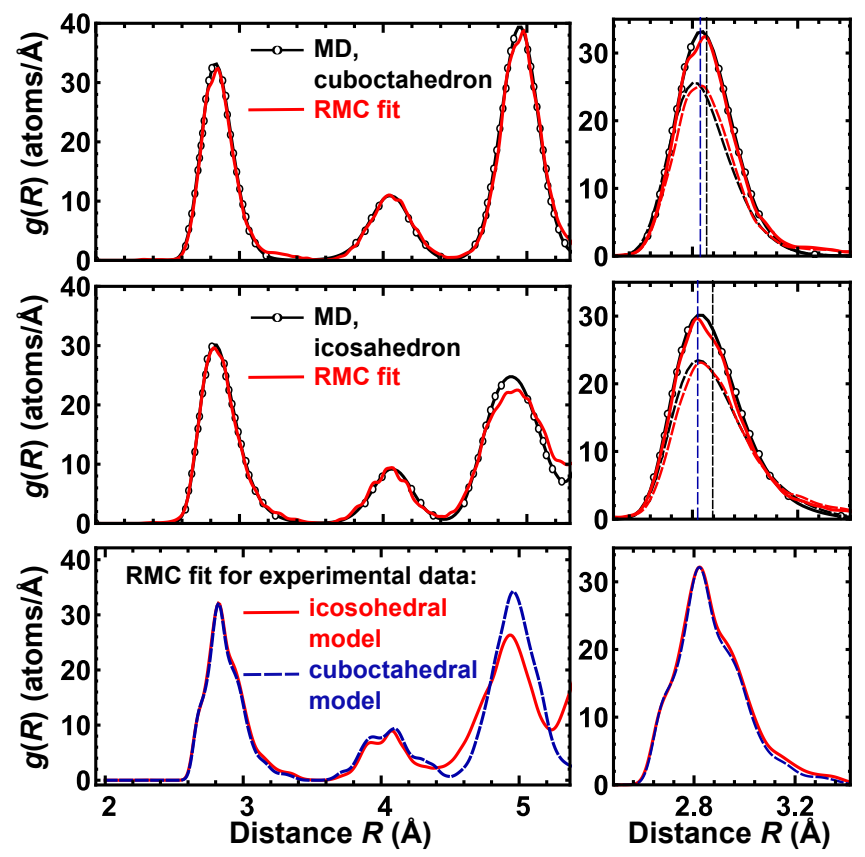

Figure 5: (Color online) Radial distribution functions $g(R)$, obtained in RMC/EA simulations using MD-EXAFS, as well as using experimental EXAFS for Au147 nanoparticles. For RDFs, which were obtained using MD-EXAFS, RDFs calculated directly from MD simulations are also shown for comparison. Dashed lines in the right panels show partial RDFs for the first coordination shell that were calculated for the surface atoms only (dashed red line results of RMC/EA simulations, dashed black line - RDF, calculated from MD coordinates). Vertical black line (long dashes) in the right panels denotes the average interatomic distance for the first coordination shell that was calculated according to equation (4). Vertical blue line (short dashes) in the right panels denotes the interatomic distance, as determined by FEFFIT analysis in harmonic approximation.

model, and both theoretical spectra are in a good agreement with experimental data. Note that empirical parameters of Sutton-Chen model were chosen to reproduce the properties of the bulk material. Hence the applicability of this model to the simulations of nanostructured material cannot be considered as granted. Therefore the observed encouraging agreement between experimental and simulated EXAFS spectra is an important argument that supports the validity of Sutton-Chen force field model for atomic scale modelling of nanosized metallic catalysts. 
in fcc lattice of bulk material. Icosahedral model, in turn, is more distorted, and even in the equilibrium there are two different interatomic distances in the first coordination shell of gold atoms in icosahedral particle: $\sim 2.80 \AA$ and $\sim 2.94 \AA$ [57]. Larger disorder of the icosahedral particle is supported also by observed lower amplitude of the main FT peaks for corresponding MD-EXAFS spectrum. From comparison of MD-EXAFS spectra with experimental one, one can conclude that the more distorted icosahedral structure is in a better agreement with experimental data. This finding is in agreement with the results of DFT and ab initio MD simulations, which suggest that for gold nanoparticles with 147 atoms icosahedral geometry is more stable [12.

When the EXAFS spectra for gold nanoparticles are compared with the ones for bulk material, one can conclude that EXAFS oscillations and amplitudes of corresponding FT peaks are strongly reduced for nanostructured material due to pronounced disorder effects. There are almost no contributions to the experimental EXAFS spectrum with corresponding distances $R>5.0 \AA$. This limits significantly the information amount, available from EXAFS analysis, especially regarding distant coordination shells. Therefore in this case it is especially important to validate the results of RMC/EA analysis using available model data.

Results of RMC/EA fitting for model MD-EXAFS data for gold nanoparticles are shown in the two upper panels of Fig. 4. As one can see, despite the larger disorder that is present in the system, and despite the fact that gold atoms now occupy positions that are not equivalent from crystallographic point of view, our RMC/EA simulations are still able to find a structure model that is in a persuasive agreement with input data. To check the accuracy of the obtained structure model, we again compare radial distribution functions (Fig. 5) and values of structure parameters (Table 3), calculated directly from 
the MD coordinates and for final atomic configuration that was obtained in RMC/EA reconstruction. Note that coordination numbers $N_{i}$ are not refined in our RMC/EA simulations, but are imposed by the geometry of starting model.

Our results suggest that even with limited information available, RMC/EA method is able to reproduce reliably the broad, asymmetric distributions of atoms within the first two coordination shells. Average interatomic distances are reproduced with accuracy about $0.005 \AA$ for the first coordination shell and about $0.01 \AA$ for the second coordination shell. MSRD factors for the first and second coordination shells are reproduced with accuracy about $0.001 \AA^{2}$ and $0.002 \AA^{2}$, respectively.

For more ordered cuboctahedral structure, the accuracy of RMC/EA analysis is comparable with the one, provided by conventional FEFFIT fitting within ${ }_{430}$ quasi-harmonic approximation. For more distorted icosahedral structure, however, the structure parameters, obtained from RMC/EA modelling, are significantly closer to the values, calculated directly from MD coordinates, than estimations from FEFFIT analysis. The inability that is shown in this work of conventional EXAFS fitting to reproduce reliably the MSRD factors for small metallic nanoparticles with strongly distorted bond lengths distribution is in agreement with the results of previous studies [9, 12. Let us emphasize here, nevertheless, that the observed disagreement between MD results and interatomic distances and disorder factors obtained by FEFFIT analysis does not imply that there is something wrong with the conventional fitting procedure. It just indicates, rather, that in the case of asymmetric distributions the terms "interatomic distance" and "width of distribution" themselves become vaguely defined. In fact, the values of interatomic distances, estimated by conventional fitting in harmonic approximation, are in a good agreement with the positions of maxima of RDFs (see the right panels in the upper two rows of Fig. 5). For 
dral MD model the distances that correspond to RDFs maxima are $2.871 \AA$, $2.832 \AA$ and $2.831 \AA$, respectively, as calculated from MD coordinates, and are close to the values estimated by conventional fitting in harmonic approximation (2.876(2) $\AA, 2.834(3) \AA$ and 2.823(5) $\AA$, respectively (see Table 2 and Table 3).

450 This finding indicates that conventional fitting tends to yield the distance corresponding to distribution maximum rather than the mean value of bond lengths distribution, as defined by equation (4). The particular strength of RMC-like approaches in this case is that as a result of the analysis one obtains a full shape of bond lengths distribution $g(R)$, from which then any statistical quantities of interest can be derived.

Another important aspect of RMC/EA simulations is that they can provide, in principle, more information than just $1 \mathrm{D}$ representation of relative atomic distribution, as given by $g(R)$ function. It is known, for instance, that the local structure around atoms at NP core and at its surface can be significantly different. For the latter, the distribution of bond lengths is expected to be even more distorted, due to additional anharmonicity of the potential for the atoms in non-symmetric surrounding 9. Therefore in Fig. 5 and Table 3 we show separately the RDF function and structure parameters for the first coordination shell, calculated for atoms at NP surface only. A reasonable agreement between RMC/EA results and corresponding quantities, calculated from MD coordinates, that was achieved in this idealized case of analysis of model data demonstrates the feasibility of RMC/EA-EXAFS studies for even more detailed investigations of the structure of nanosized catalysts.

The limitations of RMC/EA-EXAFS approach, however, are also clearly seen in Fig. 5. Significant differences in the shapes of MD-RDF and RMC/EARDF at $R$ values larger than about $4.5 \AA$ were observed, especially for the 
Table 3: Coordination numbers $N_{i}$, average interatomic distances $R_{i}$ and MSRD factors $\sigma_{i}^{2}$ for the first two coordination shells that were obtained for final RMC/EA configuration in simulations using MD-EXAFS data for Au147 nanoparticles. $N_{S}, R_{S}$ and $\sigma_{S}^{2}$ are corresponding structure parameters for the first coordination shell, calculated for atoms at the surface of nanoparticle only. For comparison, the values of structure parameters that were calculated directly from molecular dynamics coordinates are given $\left(\mathrm{MD}_{\text {cub }}\right.$ for cuboctahedral particle and $\mathrm{MD}_{\text {ico }}$ for icosahedral particle), and also the results that were obtained using conventional FEFFIT fitting within harmonic $(. . H)$ and quasi-harmonic $(. . \mathrm{QH})$ approximations. $C_{3}$ values, obtained in quasi-harmonic fit, are $3.9(6) \cdot 10^{-4} \AA^{3}$ for cuboctahedral model and $7.0(9) \cdot 10^{-4} \AA^{3}$ for icosahedral model.

\begin{tabular}{lcccc|cccc}
\hline Parameter & MD $_{\text {cub }}$ & FEFFIT $_{H}$ & FEFFIT $_{Q H}$ & RMC/EA $^{\prime}$ & MD $_{\text {ico }}$ & FEFFIT $_{H}$ & FEFFIT $_{Q H}$ & RMC/EA $^{2}$ \\
\hline$N_{1}$ & 8.98 & $8.8(2)$ & $8.9(2)$ & 8.98 & 9.47 & $7.3(6)$ & $7.7(5)$ & 9.47 \\
$R_{1}(\AA)$ & 2.855 & $2.834(3)$ & $2.856(3)$ & $2.854(2)$ & 2.877 & $2.823(5)$ & $2.860(5)$ & $2.881(2)$ \\
$\sigma_{1}^{2}\left(\AA^{2}\right)$ & 0.0114 & $0.0100(3)$ & $0.0100(2)$ & $0.0114(2)$ & 0.0160 & $0.0104(6)$ & $0.0101(3)$ & $0.017(2)$ \\
$N_{2}$ & 4.00 & - & - & 4.00 & 3.27 & - & - & 3.27 \\
$R_{2}^{2}(\AA)$ & 4.040 & - & - & $4.06(1)$ & 4.062 & - & - & $4.05(1)$ \\
$\sigma_{2}^{2}\left(\AA^{2}\right)$ & 0.0211 & - & - & $0.021(1)$ & 0.0198 & - & - & $0.024(2)$ \\
$N_{S}$ & 7.17 & - & - & 7.17 & 7.96 & - & - & 7.96 \\
$R_{S}^{2}(\AA)$ & 2.842 & - & - & $2.85(1)$ & 2.891 & - & - & $2.90(1)$ \\
$\sigma_{S}^{2}\left(\AA^{2}\right)$ & 0.0126 & - & - & $0.011(1)$ & 0.0195 & - & - & $0.019(1)$ \\
\hline
\end{tabular}

more distorted icosahedral model. This is a result of insufficient information, available from the EXAFS spectrum in this case. The fact that the peak of $\mathrm{RDF}$ that corresponds to the third coordination shell is more broader for the 475 structure, reconstructed by RMC/EA, than for the original MD structure, is in agreement with the observation that RMC-like methods tend to converge to the most distorted of all structures that are still consistent with the input data and imposed constraints [58].

Finally, we will discuss the results, obtained in the RMC/EA analysis of experimental EXAFS data for Au147 nanoparticles. In this case we have performed simulations with two different initial structure models: with icosahedral and cuboctahedral geometries. The same values for amplitude reduction factor $S_{0}^{2}$ and correction to photoelectron energy origin $\Delta E_{0}$ were used as for calculations for gold foil.

FTs of theoretical EXAFS spectra, corresponding to the final RMC/EA configuration, are compared with experimental EXAFS spectrum in bottom 
panel of Fig. 4. The representative particles from final RMC/EA configurations are shown in the insets. Since the maximal allowed displacements of atoms from their equilibrium positions were constrained to be smaller than $0.5 \AA$, the final structure models preserve the general shape of the initial structure model. Nevertheless, the calculated spectra that were obtained in simulations with two different geometries are remarkably similar, and both are equally close to the experimental spectrum. Overall agreement between the results of simulations and experiment (Fig. 4) is satisfactory and, again, is better than the one between experimental EXAFS and MD-EXAFS for Au147 system (Fig. 1). The most significant difference between experimental data and simulated spectra is visible in the shape of the left shoulder of the main peak (in the $R$ range from 1.0 to $2.0 \AA$ ), and is due to the artifacts of background subtraction procedure for experimental spectrum.

RDFs that were obtained by RMC/EA method are shown in bottom panels of Fig. 5. Taking into account that calculations with both used initial structure models resulted in very close final EXAFS spectra, it is not surprising that the first two peaks of RDF functions, corresponding to the calculations with different structure models, are also similar. Note, however, that the RDF peak, corresponding to the first coordination shell, has a slightly larger area in the case of icosahedral model due to slightly larger average number of nearest neighbors, imposed by icosahedral geometry. For the RDF peak, corresponding to the second coordination shell, the situation is opposite. Larger discrepancies between the two used structure models should be expected for the third (and further) coordination shells. However, as was demonstrated by our calculations using model Au147 data, these further coordination shells practically do not contribute to the room temperature EXAFS spectra of Au147 nanoparticles, and therefore cannot be used for the analysis. 
The inability of RMC/EA-based EXAFS analysis to distinguish between two

515 the obtained results demonstrate the robustness of RMC/EA approach. Since almost the same shape of the first RDF peaks was obtained in calculations with so different structure models, one can expect that the radial distributions of atoms, extracted from $\mathrm{RMC} / \mathrm{EA}$ simulations, will be reliable even if starting experimentally investigated sample. This is a very important conclusion, since, clearly, in most studies of nanostructured samples it is not possible to know a priori the equilibrium structure of the material.

Table 4: Coordination numbers $N_{i}$, average interatomic distances $R_{i}$ and MSRD factors $\sigma_{i}^{2}$ for the first two coordination shells in Au147 nanoparticles that were obtained from experimental $\mathrm{Au} \mathrm{L} \mathrm{L}_{3}$-edge EXAFS by RMC/EA simulations with icosahedral starting geometry, and in ab initio MD simulations 12. For completeness, the results that were obtained using conventional FEFFIT fitting of experimental data within harmonic $(. . H)$ and quasi-harmonic $(. . \mathrm{QH})$ approximations 12 are also reported. Corresponding $C_{3}$ value, obtained in quasi-harmonic fit, is $5(2) \cdot 10^{-4} \AA^{3}[12]$.

\begin{tabular}{lcccc}
\hline Parameter & FEFFIT $_{H}[12$ & FEFFIT $_{Q H}[12$ & RMC/EA & ab initio MD [12] \\
\hline$N_{1}$ & $9(1)$ & $9(1)$ & 9.47 & 9.47 \\
$R_{1}(\AA)$ & $2.816(5)$ & $2.84(1)$ & $2.881(4)$ & 2.871 \\
$\sigma_{1}^{2}\left(\AA^{2}\right)$ & $0.012(1)$ & $0.012(1)$ & $0.017(1)$ & 0.00173 \\
$N_{2}$ & - & - & 3.27 & - \\
$R_{2}^{2}(\AA)$ & - & - & $4.05(1)$ & - \\
$\sigma_{2}^{2}\left(\AA^{2}\right)$ & - & - & $0.025(1)$ & - \\
\hline
\end{tabular}

Since in this particular case EXAFS spectrum alone does not contain sufficient information, one can use the findings from other methods to distinguish between the two possible geometries. In particular, as was mentioned before, both DFT simulations 12 and our modelling using Sutton-Chen potential suggest that icosahedral geometry is more likely for Au147 (and smaller [59]) gold nanoparticles. Therefore, to conclude our discussion on the structure of Au147 nanoparticles, in Table 4 we list the values of structure parameters, obtained from our RMC/EA simulations for Au147 nanoparticles with icosahedral ge- 
ometry. Note that thus obtained values for interatomic distances and disorder factors are in a good agreement with the results of our classical MD simulations (see Table 3), but also with the results of ab initio MD simulations, reported in 535 12.

\section{Conclusions}

In this study a combination of EXAFS analysis and reverse Monte Carlo and evolutionary algorithm simulations is proposed as a powerful tool for the studies of local structure in metallic catalysts. The presented approach is very versatile and can be adapted to investigate small nanoparticles, where one expects large structural disorder and essentially asymmetric distributions of bond lengths, as well as to probe disorder effects in distant coordination shells of bulk and bulk-like materials. In particular, in this paper we have applied this method to investigate the local structure of Au147 nanoparticles. We have found that currently available experimental EXAFS spectra alone do not contain sufficient amount of information to reconstruct the 3D structure of such metallic nanoparticles unambiguously. RMC/EA approach allows us, nevertheless, to reconstruct the distribution of atoms in the nearest coordination shells reliably, especially when such simulations are complemented with the results of DFT simulations or MD modelling.

We have demonstrated that simple models, obtained in classical molecular dynamics simulations, can be successfully used to validate various approaches for EXAFS data analysis, and to estimate statistical errors of analysis, since they are able to provide realistic models for thermal disorder in the material. Moreover, we have demonstrated that for a limited number of physical problems, such as the case of bare metallic nanoparticles, classical MD simulations with Sutton-Chen force-field themselves are a powerful tool for structural investiga- 
tions, and are able to provide description of material structure and dynamics with accuracy that can compete with the one, provided by ab initio approaches.

\section{Acknowledgments}

We gratefully acknowledge support from the National Science Foundation under the DMREF program Grant No. CHE-1534184. The computational work was performed on the LASC cluster-type computer at Institute of Solid State Physics of the University of Latvia.

\section{References}

[1] P. Crespo, R. Litrán, T. Rojas, M. Multigner, J. De la Fuente, J. SánchezLópez, M. García, A. Hernando, S. Penadés, A. Fernández, Phys. Rev. Lett. 93 (2004) 087204.

[2] Y. Volokitin, J. Sinzig, L. Dejongh, G. Schmid, M. Vargaftik, I. Moiseev, Nature 384 (1996) 621-623.

[3] A. Pinchuk, U. Kreibig, A. Hilger, Surf. Sci. 557 (2004) 269-280.

[4] S. Mostafa, F. Behafarid, J. R. Croy, L. K. Ono, L. Li, J. C. Yang, A. I. Frenkel, B. R. Cuenya, J. Am. Chem. Soc. 132 (2010) 15714-15719.

[5] C. Q. Sun, Prog. Solid State Chem. 35 (2007) 1-159.

575

[6] P. Montano, G. Shenoy, E. Alp, W. Schulze, J. Urban, Phys. Rev. Lett. 56 (1986) 2076.

[7] R. Lamber, S. Wetjen, N. I. Jaeger, Phys. Rev. B 51 (1995) 10968.

[8] O. Guliamov, A. I. Frenkel, L. D. Menard, R. G. Nuzzo, L. Kronik, J. Am. Chem. Soc. 129 (2007) 10978-10979. 
[9] B. S. Clausen, J. K. Nørskov, Top. Catal. 10 (2000) 221-230.

[10] A. Witkowska, A. Di Cicco, E. Principi, Phys. Rev. B 76 (2007) 104110.

[11] A. Yevick, A. I. Frenkel, Phys. Rev. B 81 (2010) 115451.

[12] S. T. Chill, R. M. Anderson, D. F. Yancey, A. I. Frenkel, R. M. Crooks, G. Henkelman, ACS Nano 9 (2015) 4036-4042.

585 [13] A. I. Frenkel, Phys. Scripta 90 (2015) 098004.

[14] S. J. Billinge, I. Levin, Science 316 (2007) 561-565.

[15] J. J. Rehr, R. C. Albers, Rev. Mod. Phys. 72 (2000) 621.

[16] A. I. Frenkel, C. W. Hills, R. G. Nuzzo, J. Phys. Chem. B 105 (2001) 12689-12703.

[17] A. I. Frenkel, Chem. Soc. Rev. 41 (2012) 8163-8178.

[18] M. Newville, B. Ravel, D. Haskel, J. Rehr, E. Stern, Y. Yacoby, Physica B 208 (1995) 154-156.

[19] A. Frenkel, J. Rehr, Phys. Rev. B 48 (1993) 585.

[20] G. Dalba, P. Fornasini, J. Synchrotron Red. 4 (1997) 243-255.

${ }_{595}$ [21] A. Di Cicco, M. Minicucci, E. Principi, A. Witkowska, J. Rybicki, R. Laskowski, J. Phys.: Condens. Matter 14 (2002) 3365.

[22] A. Filipponi, J. Phys.: Condens. Mattter 13 (2001) R23.

[23] A. Kuzmin, Physica B 208-209 (1995) 175-176.

[24] A. Cintins, A. Anspoks, J. Purans, A. Kuzmin, J. Timoshenko, P. Vladimirov, T. Gräning, J. Hoffmann, IOP Conf. Ser.: Mater. Sci. Eng. 77 (2015) 012029. 
[25] A. I. Frenkel, J. Synchrotron Rad. 6 (1999) 293-295.

[26] B. Prasai, A. Wilson, B. Wiley, Y. Ren, V. Petkov, Nanoscale 7 (2015) $17902-17922$.

605

610

[27] D. F. Yancey, L. Zhang, R. M. Crooks, G. Henkelman, Chem. Sci. 3 (2012) 1033-1040.

[28] R. M. Anderson, L. Zhang, J. A. Loussaert, A. I. Frenkel, G. Henkelman, R. M. Crooks, ACS nano 7 (2013) 9345-9353.

[29] R. M. Anderson, D. F. Yancey, L. Zhang, S. T. Chill, G. Henkelman, R. M. Crooks, Accounts Chem. Res. 48 (2015) 1351-1357.

[30] D. F. Yancey, S. T. Chill, L. Zhang, A. I. Frenkel, G. Henkelman, R. M. Crooks, Chem. Sci. 4 (2013) 2912-2921.

[31] O. M. Roscioni, N. Zonias, S. W. Price, A. E. Russell, T. Comaschi, C.-K. Skylaris, Physical Review B 83 (2011) 115409.

[32] S. W. Price, N. Zonias, C.-K. Skylaris, T. I. Hyde, B. Ravel, A. E. Russell, Phys. Rev. B 85 (2012) 075439.

[33] R. McGreevy, L. Pusztai, Mol. Simul. 1 (1988) 359.

[34] R. L. McGreevy, P. Zetterstrm, J. Non-Cryst. Solids 293-295 (2001) 297 303.

[35] J. Timoshenko, A. Kuzmin, J. Purans, J. Phys.: Condens. Matter 26 (2014) 055401.

[36] J. Timoshenko, A. Anspoks, A. Kalinko, A. Kuzmin, Acta Mater. 79 (2014) 194-202.

[37] J. Timoshenko, A. Anspoks, A. Kalinko, A. Kuzmin, Phys. Status Solidi A 212 (2015) 265-273. 
[38] R. M. Crooks, M. Zhao, L. Sun, V. Chechik, L. K. Yeung, Accounts Chem. Res. 34 (2001) 181-190.

[39] N. Metropolis, A. W. Rosenbluth, M. N. Rosenbluth, A. H. Teller, E. Teller, J Chem. Phys. 21 (1953) 1087-1092.

${ }_{630}$ [40] S. Kirkpatrick, C. D. Gelatt, M. P. Vecchi, Science 220 (1983) 671.

[41] J. Holland, Adaptation in natural and artificial systems: an introductory analysis with applications to biology, control, and artificial intelligence, MIT Press Cambridge, MA, USA, 1992.

[42] A. E. Eiben, J. Smith, Nature 521 (2015) 476-482.

${ }_{635}$ [43] J. Timoshenko, A. Anspoks, A. Kalinko, A. Kuzmin, Z. Phys. Chem. (2015).

[44] A. Ankudinov, B. Ravel, J. Rehr, S. Conradson, Phys. Rev. B 58 (1998) 7565.

[45] H. Funke, A. Scheinost, M. Chukalina, Phys. Rev. B 71 (2005) 094110.

[46] J. Timoshenko, A. Kuzmin, Comp. Phys. Commun. 180 (2009) 920.

[47] J. Timoshenko, A. Kuzmin, J. Purans, Comp. Phys. Commun. 183 (2012) 1237.

[48] A. Sutton, J. Chen, Phil. Mag. Lett. 61 (1990) 139-146.

[49] J. D. Gale, Phil. Mag. B 73 (1996) 3.

${ }_{645}$ [50] J. D. Gale, A. L. Rohl, Mol. Simul. 29 (2003) 291.

[51] W. G. Hoover, Phys. Rev. A 31 (1985) 1695.

[52] R. W. Hockney, Methods Comput. Phys. 9 (1970) 136. 
[53] B. J. Palmer, D. M. Pfund, J. L. Fulton, J. Phys. Chem. 100 (1996) 1339313398.

${ }_{650}^{6}$ [54] G. Ferlat, J.-C. Soetens, A. S. Miguel, P. A. Bopp, J. Phys.: Condens. Matter 17 (2005) S145.

[55] A. Frenkel, E. Stern, A. Voronel, M. Qian, M. Newville, Phys. Rev. Lett. $71(1993) 3485$.

[56] G. Dalba, P. Fornasini, R. Grisenti, J. Purans, Phys. Rev. Lett. 82 (1999) 4240.

[57] D. Glasner, A. I. Frenkel, AIP Conf. Proc. 882 (2007) 746-748.

[58] M. G. Tucker, D. A. Keen, M. T. Dove, A. L. Goodwin, Q. Hui, J. Phys.: Condens. Matter 19 (2007) 335218.

[59] G. Periyasamy, E. Durgun, J.-Y. Raty, F. Remacle, J. Phys. Chem. C 114 (2010) 15941-15950. 


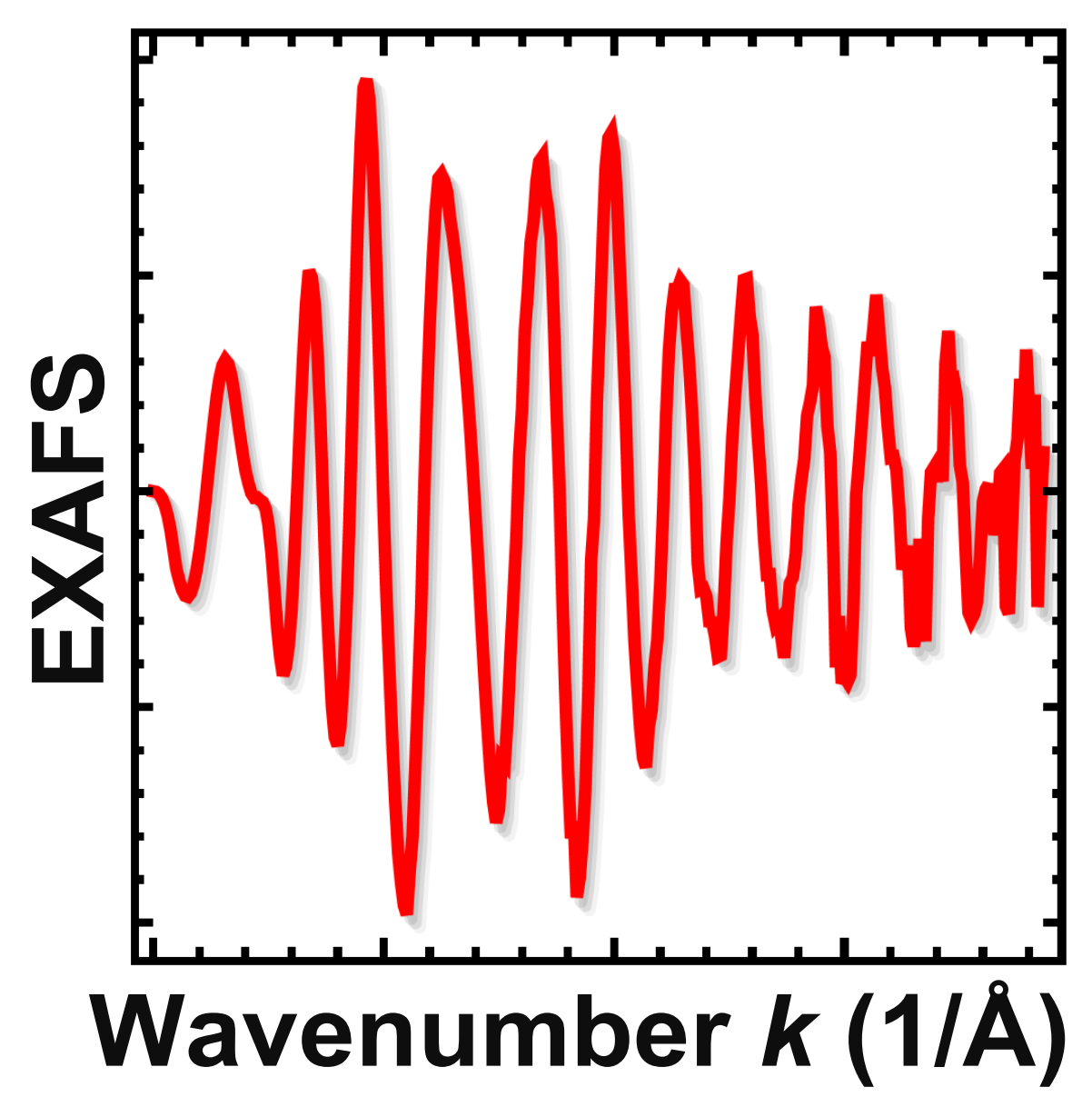

Experimental data

\section{Disordered nanoparticle}

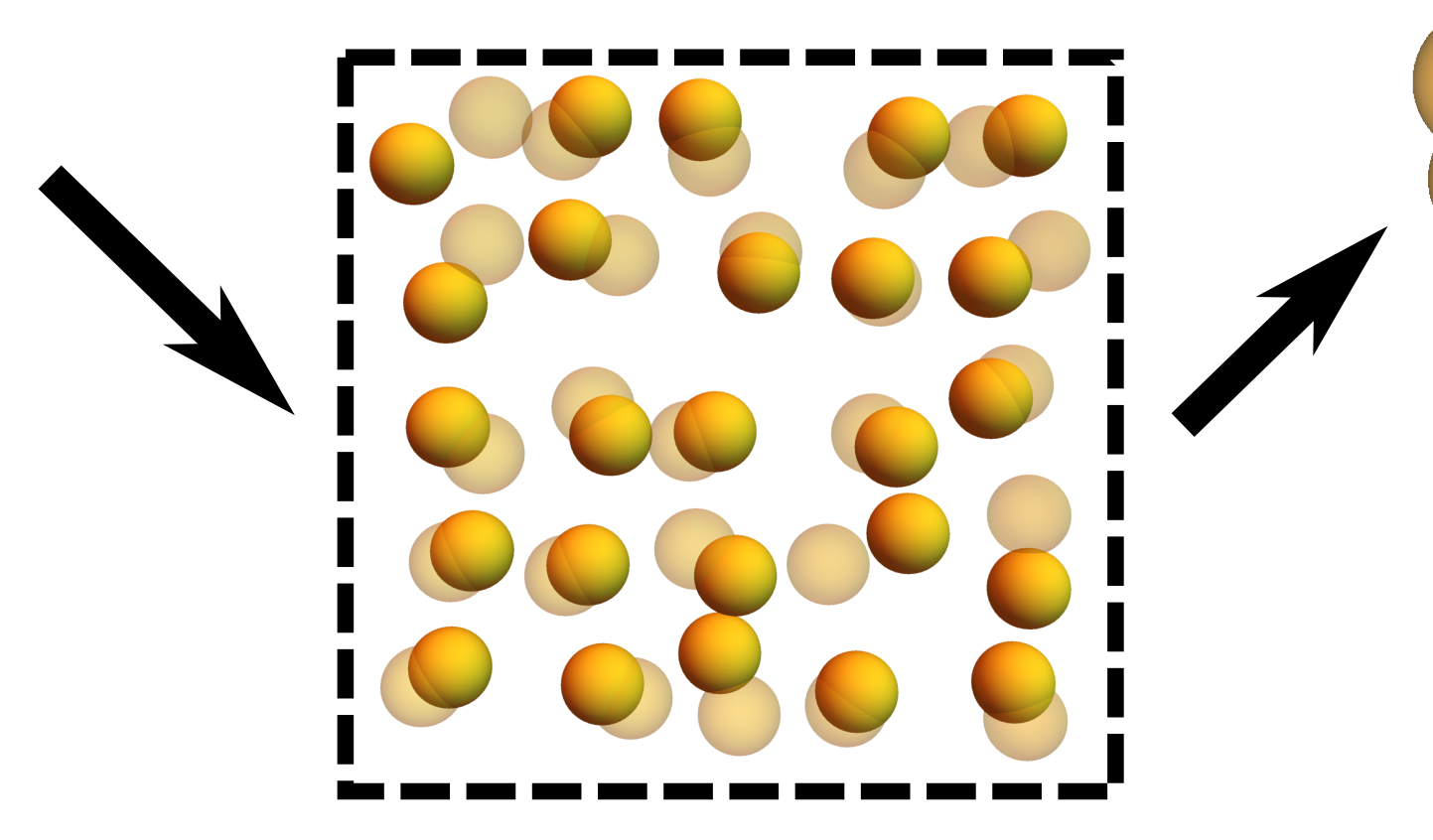

3D structure RMC simulations model 